\title{
Inflammation as a risk factor for myocardial infarction
}

\begin{abstract}
Myocardial infarction (MI) is one of the common diseases whose pathogenesis includes genetic factors. To reveal genetic backgrounds of this clinically heterogeneous disorder, we started our case-control association study by examining large-scale gene-based single nucleotide polymorphism (SNP) sets for $\sim 1,000$ patients and controls. As a core genotyping method, a combination of multiplex PCR and Invader method was used, and after genotyping $\sim 65,000$ SNPs, we found two SNPs located within lymphotoxin- $\alpha$ ( $L T A)$ gene showing significant association with MI. LTA is one of the cytokines produced in the early stages of vascular inflammatory processes. These SNPs seem to be involved in inflammation by both qualitatively and quantitatively modifying the function of LTA protein, thereby conferring a risk of MI. The genetic association was further confirmed by other researchers using white European trios. To further understand the roles of LTA protein in the pathogenesis of MI, we searched for proteins that interact directly with LTA protein and identified galectin2 protein as a binding partner of LTA protein. It is a member of galactose-binding lectin family whose function has not been well characterized. Genetic association study again revealed that an SNP in LGALS2 encoding galectin-2 was also associated with susceptibility to MI. This genetic substitution seemed to affect the transcriptional level of galectin-2, which led to altered secretion of LTA, thereby affecting the degree of inflammation. Thus, our findings indicate the importance of inflammation, especially the LTA cascade, in the pathogenesis of MI. Also, combined strategy of genetic and molecular-cellular biological approaches may be useful for clarification of the pathogenesis of common diseases in general.
\end{abstract}

T. Tanaka $(\bowtie) \cdot$ K. Ozaki

Laboratory for Cardiovascular Diseases,

Research Group for Disease-Causing Mechanisms,

SNP Research Center, Riken, 4-6-1 Shirokanedai,

Minato-ku, Tokyo 108-8639, Japan

E-mail: toshitan@ims.u-tokyo.ac.jp

Tel.: + 81-35449-5675

Fax: $+81-35449-5674$
Keywords SNP (single nucleotide polymorphism) ·

Association study · Myocardial infarction .

Inflammation

\section{Introduction}

Coronary artery diseases including myocardial infarction (MI) continue to be the principal cause of death in many countries despite changes in lifestyle and the development of new pharmacologic approaches (Breslow 1997; Braunwald 1997), indicating the importance of identifying genetic and environmental factors in the pathogenesis of these diseases to provide better treatment to patients.

MI is a disease of the vessel that feeds the cardiac muscle, called the coronary artery. Abrupt occlusion of the coronary artery results in irreversible damage to cardiac muscle. Plaque rupture with thrombosis is a well-established critical factor in the pathogenesis of MI (Falk et al. 1995; Libby 1995). Although detailed mechanisms of plaque rupture are unknown, inflammation is thought to play an important role in its pathogenesis (Ross 1999). Inflammatory mediators like cytokines are involved in atheroma formation; rapid evolution of the atheromatous injury, leading to rupture of the plaque; and intraluminal thrombosis (Ross 1999).

Epidemiological studies have revealed several risk factors for MI, including diabetes mellitus, hypercholesterolemia, and hypertension. Although each of the risk factors should have genetic components, a positive family history of MI is an independent predictor, which suggests it has a genetic background of its own (Marenberg et al. 1994). To reveal genetic backgrounds of MI, investigation through common genetic variants should be of great use since they are widely thought to contribute significantly to genetic risks of common diseases (Collins et al. 1997; Lander 1996; Risch and Merikangas 1996). Naturally, comprehensive analyses, rather than the candidate gene approach, have much 
more power to detect genetic factors in that, in theory, they can examine a whole set of genetic variations. Among the variations in the human genome, single nucleotide polymorphisms (SNP) have recently attracted attention. SNP is the most common variations and is usually biallelic. These characteristics provide a greater probability of identifying the associated variations directly if comprehensive analysis is possible, and their binary nature is also of great help in constructing a high-throughput genotyping system.

In this mini-review, we focus on our genetic association results and show that our initial hypothesis-free strategy unexpectedly revealed the importance of inflammation in the pathogenesis of MI.

\section{Identification of susceptibility locus for MI through large-scale gene-based SNP association study}

As a first step in our comprehensive association study, we genotyped 94 MI patients with our high-throughput multiplex PCR-Invader assay method (Ohnishi et al. 2001) using 92,788 gene-based SNPs (Haga et al. 2002) and compared the results with the allelic frequencies found in 658 members of the general Japanese population. We think our screening system is superior to others in two major ways. First, our SNP database (Haga et al. 2002, http://snp.ims.u-tokyo.ac.jp) was an extensive catalogue of SNPs located within exons, introns and promoter regions. Since we screened selected regions using these genebased SNPs, we could efficiently examine SNPs that might be associated with susceptibility to common diseases. Second, based on a combination of multiplex PCR and Invader assay, our method allows high-throughput, lowcost screening that requires as little as $0.1 \mathrm{ng}$ of genomic DNA per single SNP (Ohnishi et al. 2001).

The success rate for this genotyping was $70.8 \%$ (65,671 SNPs), and distribution of the allelic frequency was largely even (Ozaki et al. 2002). Characteristics of these 65,671 SNPs are summarized in Table 1 . The cutoff $P$ value of 0.01 for association in either recessive or dominant models was used for this screening, and as shown in Table 2, approximately $99 \%$ of the SNP loci that were successfully genotyped were excluded from the second screening. We further genotyped SNPs that showed $P$ value less than 0.01 in a larger replication panel of MI patient and control subjects and found that most of the loci eventually showed the lack of association at this screening phase of the project. From these observations, we think one should carefully handle the association results using fewer $(\sim 100)$ samples for common diseases. However, when one SNP (intron 1; $252 \mathrm{~A}>\mathrm{G})$ in the lymphotoxin- $\alpha(L T A)$ gene on chromosome $6 \mathrm{p} 21$ (HLA region), which was associated with MI in the screening $\left(\chi^{2}=9.4, P=0.0022\right)$, was verified, the association became much more significant, with a $\chi^{2}$ value of $18.0(P=0.000022$; homozygotes for the minor allele versus others) and an odds ratio of 1.69 [95\% confidence interval (CI): 1.32-2.15; Table 3].
Table 1 Summary of genotyped single nucleotide polymorphisms (SNPs)

\begin{tabular}{lr}
\hline Genes screened & 13,738 \\
\hline Exon & 11,694 \\
Synonymous & 1,493 \\
Non-synonymous & 1,491 \\
Non-coding region & 8,710 \\
Intron & 43,910 \\
5, flanking region & 2,038 \\
3, flanking region & 1,428 \\
Other regions $^{\mathrm{a}}$ & 6,601 \\
Total $^{\text {Tol }}$ & 65,671
\end{tabular}

${ }^{a}$ Not mapped within gene regions

The table is taken from Ozaki et al. (2002)

Then we examined whether the SNP in LTA was related to conventional risk factors of $\mathrm{MI}$, including diabetes (DM), hypertension (HT) and hyperlipidaemia (HL). We examined the genotype frequencies in the presence or absence of each risk factor in 1,133 MI samples, and found that an association between each of the three risk factors and SNP at $L T A$ was not statistically significant in a comparison of homozygote for the minor allele versus others $(P=0.14,0.96$ and 0.68 for DM, HT and HL, respectively). Thus, the association of LTA was shown to be independent of each of these conventional risk factors.

We also investigated the possibility that population stratification might be influencing the results. We examined the distribution of chi-square values for all SNP loci where chi-square test was appropriate. The mean and $95 \%$ upper bound for the values were 1.05 and 4.03 for recessive-inheritance model, respectively, and 1.04 and 3.96 for dominant-inheritance model, respectively, indicating that there was no population stratification. These analyses indicated that a gene conferring susceptibility to MI itself was present within this region.

\section{Linkage disequilibrium and haplotype structure at $L T A$ locus}

We constructed a high-density SNP map for LD mapping by direct sequencing of DNA by screening approximately $130 \mathrm{~kb}$ of the relevant region on $6 \mathrm{p} 21$, which included several other genes encoding molecules

Table 2 Distribution of $P$ value in the screening of 94 cases and 658 controls

\begin{tabular}{lcc}
\hline$P$ value & \multicolumn{2}{c}{ Number of single nucleotide polymorphisms } \\
\cline { 2 - 3 } & Dominant association & Recessive association \\
\hline$>0.01$ & 64,898 & 64,822 \\
$<1 \times 10^{-2}$ & 672 & 715 \\
$<1 \times 10^{-3}$ & 88 & 110 \\
$<1 \times 10^{-4}$ & 12 & 18 \\
$<1 \times 10^{-5}$ & 1 & 5 \\
$<1 \times 10^{-6}$ & 0 & 1 \\
Total & 65,671 & 65,671
\end{tabular}

The table is taken from Ozaki et al. (2002) 
Table 3 Association between myocardial infarction (MI) and single nucleotide polymorphisms in $L T A$

Nucleotide numbering starts from the first nucleotide of exon 1

The table is taken from Ozaki et al. (2002)

\begin{tabular}{lclll}
\hline Genotype & MI $(n=1,133)$ & Control $(n=1,006)$ & $\chi^{2}(P$ value $)$ & Odds ratio (95\% CI) \\
\hline LTA exon 1 & $10 \mathrm{G}>\mathrm{A}$ & & & \\
GG & $416(36.7 \%)$ & $378(37.6 \%)$ & AA vs GG + GA & $1.78(1.39-2.27)$ \\
GA & $504(44.5 \%)$ & $512(50.9 \%)$ & 21.6 & \\
AA & $213(18.8 \%)$ & $116(11.5 \%)$ & $(0.0000033)$ & \\
LTA intron 1 & $252 \mathrm{~A}>\mathrm{G}$ & & & \\
AA & $413(36.5 \%)$ & $371(36.9 \%)$ & GG vs AA + AG & $1.69(1.32-2.15)$ \\
AG & $511(45.1 \%)$ & $516(51.3 \%)$ & 18 & \\
GG & $209(18.4 \%)$ & $119(11.8 \%)$ & $(0.000022)$ & \\
LTA exon 3 & $804 \mathrm{C}>\mathrm{A}, \mathrm{T} 26 \mathrm{~N}$ & & & \\
CC & $414(36.5 \%)$ & $376(37.4 \%)$ & AA vs CC $+\mathrm{CA}$ & $1.78(1.39-2.27)$ \\
CA & $506(44.7 \%)$ & $514(51.1 \%)$ & 21.6 & \\
AA & $213(18.8 \%)$ & $116(11.5 \%)$ & $(0.0000033)$ & \\
\hline
\end{tabular}

related to inflammation, such as TNF, LTA, NFKBIL1, and BATl. We found 26 SNPs with frequencies high enough to make them relevant to the search for genes predisposing to common diseases ( $>25 \%$ of minor alleles), and consequently found one extended block of intense LD. We further genotyped these 26 SNPs by expanding the sample size. Although most of them showed no significant association with the MI phenotype, three of these SNPs revealed a tight association with MI when we compared frequencies of homozygosity for the minor allele between cases and controls (Table 3). We further confirmed association with MI and $L T A$ exon3 $804 \mathrm{C}>\mathrm{A}$ SNP using increased sample sizes $(2,833$ cases and 3,399 control subjects) and obtained similar association results $(P=0.0000014$, recessive association model; Table 4). Furthermore, a recent transmission disequilibrium test (TDT) analysis of 447 trio families with coronary artery disease (CAD) in white Europeans (PROCARDIS) demonstrated that the LTA $804 \mathrm{C}$ allele (26N-LTA) was excessively transmitted to affected offspring $\left(\chi^{2}=8.44, P=0.0018, \gamma^{2}=1.96\right.$, PROCARDIS Consortium 2004), indicating the generality of this variation in the pathogenesis of MI. Recently, other Japanese researchers have claimed the lack of association (Yamada et al. 2004); we think there is one flaw in their paper. The genotype frequencies of our MI group and their MI group were almost the same, and the difference was in control groups. Their control group included only those who had at least one coronary risk factor, which created a risk of sampling biases. Also, several other studies have examined this locus, and their genotype frequencies were similar to our control groups (Nishimura et al. 2000; Quasney et al. 2001; Tokushige et al. 2003; Iwanaga et al. 2004). Thus, it might be natural to consider that the different association results were due to the unique characteristics of their control group, not ours.

\section{Function of intron1 SNP in LTA}

To determine whether the two SNPs in the LTA gene, $10 \mathrm{G}>\mathrm{A}$ in exon 1 and $252 \mathrm{~A}>\mathrm{G}$ in intron 1 , would affect its expression level, we constructed three kinds of plasmids with a genomic fragment containing both SNPs (10G-252A, 10A-252G, and 10A-252A haplotypes, respectively) upstream of a luciferase gene transcriptional unit. We did not investigate a construct containing $10 \mathrm{G}-252 \mathrm{G}$ because this haplotype was not present in the individuals we examined. As shown in Fig. 1a, the clone containing the 10A-252G haplotype showed 1.5-fold greater transcriptional activity than clones containing the other two haplotypes, indicating that the substitution in intron 1 , but not the one in exon 1, affected transcription of the $L T A$ gene. We also used reporter-gene assays to investigate potential effects of SNPs in the promoter regions of the IKBL and $B A T I$ genes, and found that a $-63 \mathrm{~A}$ allele of $I K B L$ might cause a moderate reduction in transcriptional activity (Fig. 1b).

Furthermore, since any known DNA-binding proteins could probably bind to parts of the DNA sequence containing the SNP in intron 1 of $L T A$, we examined whether a nuclear extract from Jurkat cells was able to bind to oligonucleotides corresponding to genomic sequences that included $252 \mathrm{~A}$ or $252 \mathrm{G}$ alleles. As shown in Fig. 1c, the band that appeared when we used the oligonucleotide corresponding to the $G$ allele
Table 4 Confirmation of association between myocardial infarction (MI) and lymphotoxin- $\alpha(L T A)$ gene single nucleotide polymorphisms

\begin{tabular}{lcllll}
\hline Genotype & MI $(\%)$ & Control $(\%)$ & $\chi^{2}(P$ value $)$ & \multicolumn{2}{l}{ HWE- $\chi^{2}$ test $(P)$} \\
\cline { 5 - 6 } & & & MI & Control \\
\hline Exon 3 & $804 \mathrm{C}>$ A, T26N & & & \\
CC & $1,028(36.3)$ & $1,333(39.2)$ & AA vs CC + CA & $3.34(0.068)$ & $3.25(0.072)$ \\
CA & $1,318(46.5)$ & $1,630(48.0)$ & 23.31 & \\
AA & $487(17.2)$ & $436(12.8)$ & $(0.0000014)$ & \\
Total & 2,833 & 3,399 & &
\end{tabular}


Fig. 1a-c Modification of transcriptional activity by lymphotoxin- $\alpha(L T A)$ gene single nucleotide polyphormisms (SNPs) (Ozaki et al. 2002). Transcriptional regulatory activity affected by SNPs in a intron $1(252 \mathrm{~A}>\mathrm{G})$ of $L T A$ and $\mathbf{b}$ the promoter region $(-63 \mathrm{~T}>\mathrm{A})$ of NFKBIL1. Each experiment was performed independently three times, with each sample measured in triplicate. ${ }^{*} P<0.01$ in comparison between $10 \mathrm{G}$ $252 \mathrm{~A}$ and $10 \mathrm{~A}-252 \mathrm{G}$ haplotypes; $* * P<0.01$ in comparison between $-63 \mathrm{~A}$ allele and -63 T allele (Student's $t$ test). c Binding of unknown nuclear factor(s) to intron 1 of $L T A$. An arrow indicates the band showing tighter binding of nuclear factor(s) to the G-allele. The figure is taken from Ozaki et al. (2002)
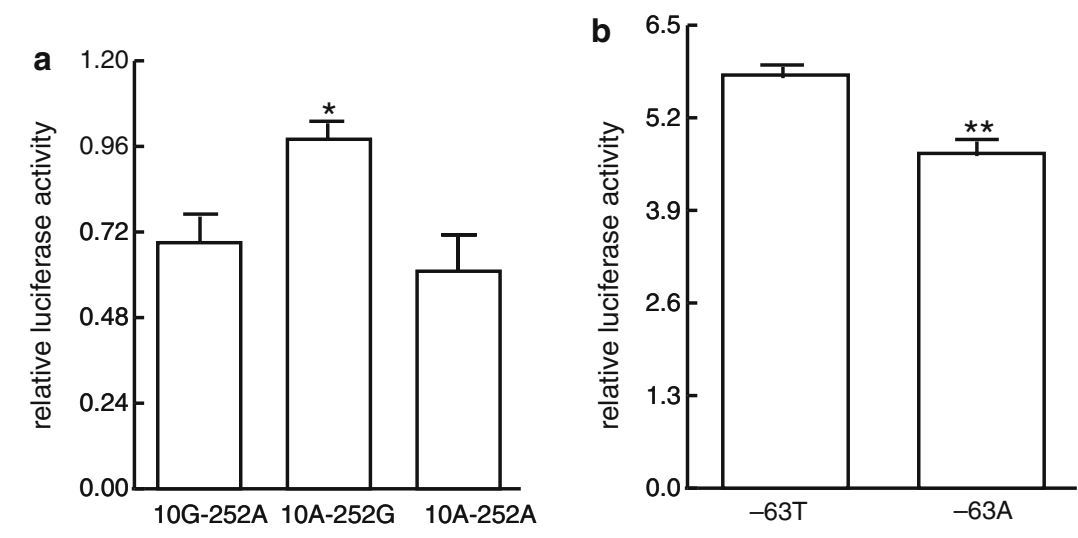

\begin{tabular}{lcccccc} 
C & & & & & \\
labeled oligo & & A-allele & & \multicolumn{3}{c}{ G-allele } \\
\hline competitor & - & - & + & - & - & + \\
\hline extract & - & + & + & - & + & + \\
\hline
\end{tabular}

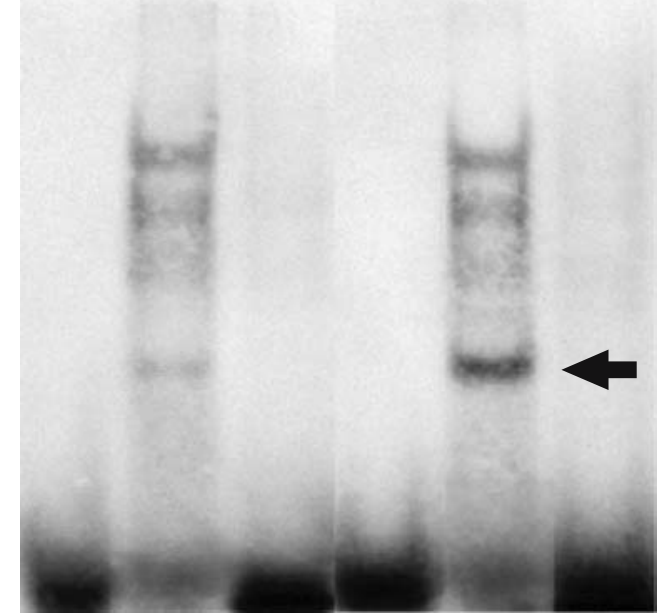

was more intense than the band corresponding to the A allele, indicating that some nuclear factor present in Jurkat cells was binding more tightly to the $G$ allele than to the A allele. This result raised the possibility that unidentified nuclear factor(s) regulating transcription of $L T A$ by binding to this region may represent novel molecular entities related to $\mathrm{MI}$ susceptibility.

\section{T26N variation in LTA protein}

In addition to SNPs in the putative promoter or transcriptional-regulator sequences of the LTA gene, we found one SNP that would substitute asparagine for threonine at codon $26(\mathrm{~T} 26 \mathrm{~N})$. The LTA product can induce adhesion molecules and cytokines from vascular endothelial cells, vascular smooth-muscle cells, and several kinds of leukocytes, as its contribution to the inflammatory process (Libby 1995; Ross 1999). To address whether these biological activities could be influenced by the amino-acid substitution in the gene product, we constructed expression vectors containing each allele, and purified mature recombinant LTA proteins in Escherichia coli. The ability of each allele to induce expression of adhesion molecules and cytokines was examined in cultured human coronary artery endothelial cells (HCAEC) and in cultured human coronary artery smooth-muscle cells (HCASMC). Both types of LTA protein stimulated mRNA expression of vascular cell adhesion molecule-1 (VCAM-1), intracellular cell adhesion molecule-1 (ICAM-1), E-selectin, TNF- $\alpha$, interleukin- $1 \alpha$ (IL- $1 \alpha)$, and interleukin- $1 \beta$ (IL-1 $\beta$ ) within $4 \mathrm{~h}$, and we observed no difference in transcriptional activity when we used HCAEC. However, in HCASMC cells, 26N-LTA revealed a two-fold higher level of transcriptional activity for VCAM-1 and E-selectin than 26T-LTA (Fig. 2).

Thus, it seems that increased level of functionally modified LTA protein is associated with increased degree of inflammation, thereby conferring risk of MI (Fig. 6). 
Fig. 2 Differing abilities of $26 \mathrm{~N}$ - and 26T-lymphotoxin- $\alpha$ $(L T A)$ to induce mRNA expression of adhesion molecules (Ozaki et al. 2002). Relative mRNA levels are indicated for a VCAM1 and b SELE in HCASMC following treatment of each cell with medium only (white bars), 26TLTA (gray bars) or 26N-LTA (black bars) $(20 \mathrm{ng} / \mathrm{ml})$ for $4 \mathrm{~h}$. The figure is taken from Ozaki et al. (2002)

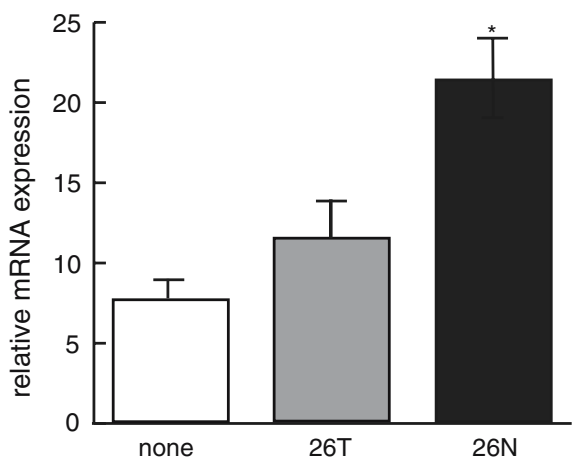

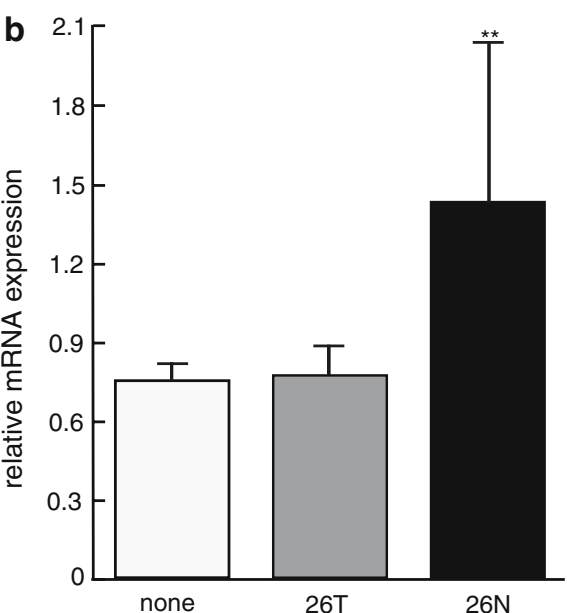

\section{Identification of galectin-2 as a binding partner of LTA}

After identification of $L T A$ as a novel genetic risk factor for myocardial infarction, we searched for proteins that interact with LTA, to better understand the role of LTA in the pathogenesis of this disease. With both the E. Coli two-hybrid system and phage display method, we could identify a protein, galectin-2, as a binding partner of LTA (data not shown). We purified both recombinant proteins raised by bacterial expression system, and direct binding between galectin-2 and LTA was tested using in vitro binding assay. As shown in Fig. 3a, galectin-2 bound directly to recombinant LTA. We further examined whether the interaction took place in mammalian cells with constructs encoding FLAG-tagged LTA and S-tagged galectin-2. Western blot analysis showed that galectin-2 was co-immunoprecipitated with LTA (Fig. 3b). Using antibodies that specifically recognize each protein, we also investigated subcellular localization of endogenous galectin-2 and LTA proteins in U937 cells and found that these proteins co-localize in the cytoplasm (Fig. 3c).

\section{Association of intron 1 SNP in LGALS2 with myocardial infarction}

Since galectin-2 was shown to bind to LTA, we next examined whether the variation(s) in $L G A L S 2$ (encoding galectin-2) were also associated with susceptibility to myocardial infarction. By re-sequencing of the LGALS2 genomic region using $32 \mathrm{MI}$ samples, we found 17 SNPs (Table 5). We then compared genotype frequencies of approximately 600 individuals with MI and 600 controls for these SNPs and found one SNP $(3279 \mathrm{C}>\mathrm{T})$ in intron1 of $L G A L S 2$ showed significant association with MI (Table 5). No particular haplotype showed higher statistical significance for association with MI than the significant SNP alone. We further confirmed this association by increasing the number of samples up to 2,302 for patients with MI and 2,038 for controls. Since minor allele frequency of the SNP was lower in the group of patients (Table 6), we concluded that the minor variant has a protective role against the risk of MI. To examine a

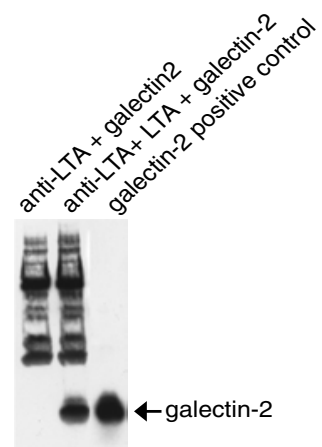

b

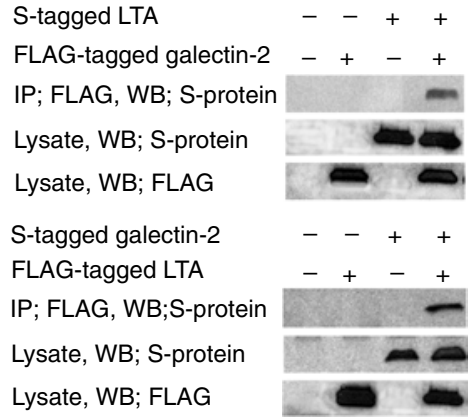

C
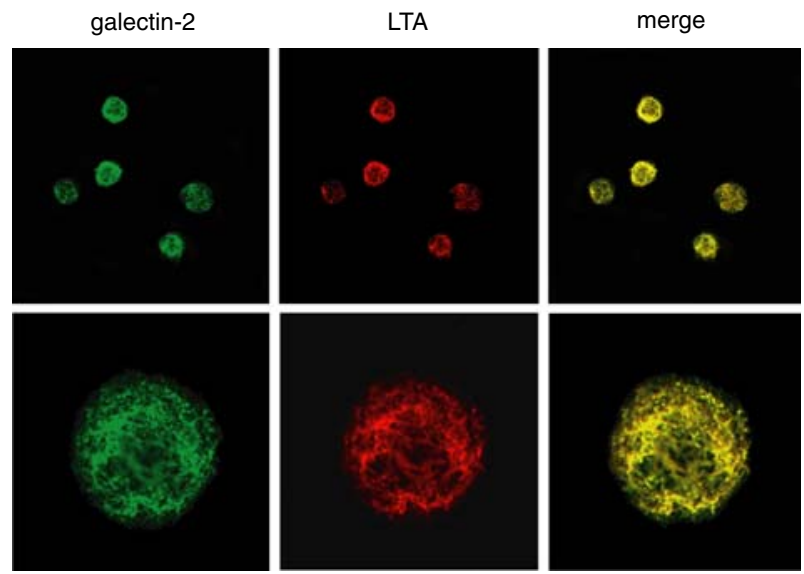

Fig. 3a-c Lymphotoxin- $\alpha(L T A)$ binds to galectin-2 (Ozaki et al. 2004). a In vitro binding assay. b Co-immunoprecipitation of tagged LTA and galectin-2 in COS7 cells. For immunoprecipitation $(I P)$ and western blot $(W B)$, anti-FLAG tag antibody agarose and S-protein horseradish peroxidase (S-protein) were used, respectively. c Co-localization of endogenous LTA with galectin-2 in U937 cells. Bottom panels are enlarged images of representative cells in the upper panels. The figure is taken from Ozaki et al. (2004) 


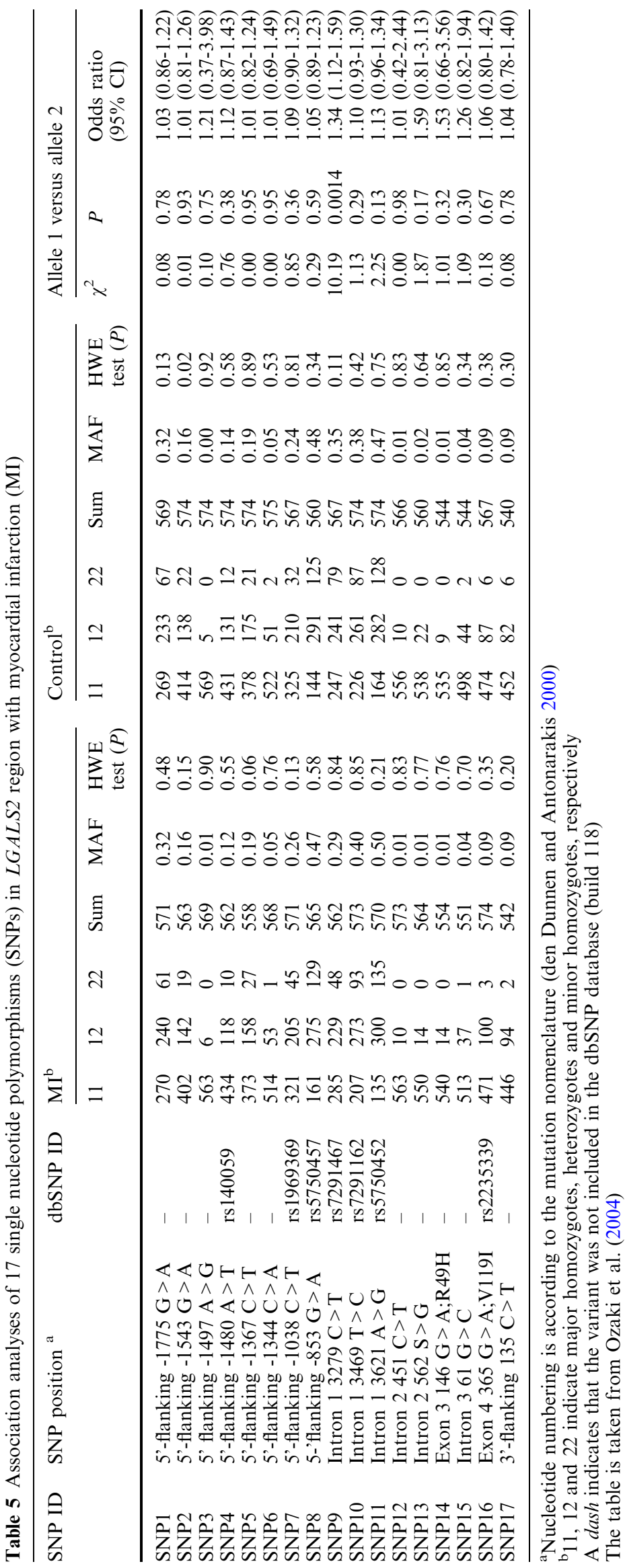


Table 6 Association of a single nucleotide polymorphism (SNP) in $L G A L S 2$ with myocardial infarction (MI)

\begin{tabular}{|c|c|c|c|c|}
\hline \multirow{2}{*}{\multicolumn{2}{|c|}{ Genotype MI }} & \multirow[t]{2}{*}{ Control } & \multicolumn{2}{|c|}{ Allele $C$ versus allele $T$} \\
\hline & & & $\begin{array}{l}\chi^{2} \\
(P \text { value })\end{array}$ & $\begin{array}{l}\text { Odds ratio } \\
(95 \% \mathrm{CI})\end{array}$ \\
\hline \multicolumn{5}{|c|}{ Intron $13279 \mathrm{C}>\mathrm{T}^{\mathrm{a}}$} \\
\hline $\mathrm{CC}$ & $1,077(46.8 \%)$ & $856(42.0 \%)$ & 22.1 & 1.57 \\
\hline $\mathrm{CT}$ & $1,014(44.0 \%)$ & $903(44.3 \%)$ & $(0.0000026)$ & $(1.30-1.90)$ \\
\hline TT & $211(9.2 \%)$ & $279(13.7 \%)$ & & \\
\hline Total & $2,302(100 \%)$ & $2,038(100 \%)$ & & \\
\hline
\end{tabular}

a Nucleotide numbering is according to the mutation nomenclature (den Dunnen and Antonarakis 2000)

The table is taken from Ozaki et al. (2004)

whether the intron1 SNP in $L G A L S 2$ would affect its expression level, we constructed reporter plasmids with a genomic fragment containing the SNP in the downstream of a luciferase gene and SV40 enhancer sequence. The clone containing 3279T allele showed two-fold less transcriptional activity than clones containing the 3279C allele and vector only (Fig. 4 a, b), indicating that the substitution repressed the transcriptional level of galectin-2.

\section{Function of galectin-2 protein}

Although galectin-2 was thought to be a member of the carbohydrate-binding lectin family based on sequence similarity (Gitt et al. 1992), its function was largely uncharacterized. As several members of the galectin family are known to be secreted (Hughes 1999), we first investigated the possibility of whether galectin-2 was also secreted, using $\mathrm{HeLa}$ or HepG2 cell lines transfected with $C$-terminal FLAG- or $N$-terminal Myc-tagged galectin-2 expression vector. However, we could not detect galectin-2 in culture media after transfection for 24 and $48 \mathrm{~h}$, even by concentrating the FLAG- or Myctagged protein using the corresponding anti-tag antibody agaroses (Fig. 4c, d), indicating that galectin-2 predominantly functions as an intracellular protein, although this remains to be clarified using stably transformed cell lines. Since susceptibility to SNP in $L G A L S 2$ was shown to affect expression level of galectin-2, we hypothesized that intracellular amounts of galectin-2 might regulate the secretion level of LTA, thus influencing the degree of inflammation. To clarify this, we examined changes in the secreted level of LTA caused by quantitative alteration of galectin-2, using siRNA technique. One siRNA for galectin-2 was shown to specifically repress $\sim 80 \%$ of galectin- 2 mRNA (Fig. $4 \mathrm{e}$ ) and to significantly inhibit the secretion of LTA (Fig. 4f). As shown in Fig. 4g, LTA mRNA level did not change by knockdown of galectin-2.

To investigate the regulatory mechanism of LTA secretion by galectin-2, we searched for intracellular molecules that associate with galectin-2 using the tandem affinity purification (TAP) system (Rigaut et al.

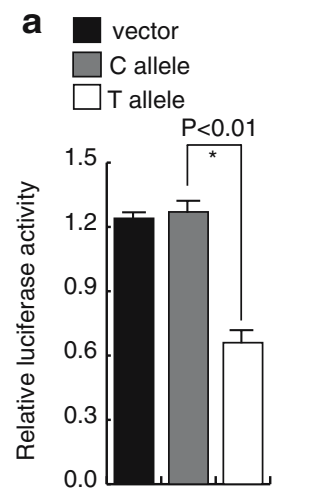

b
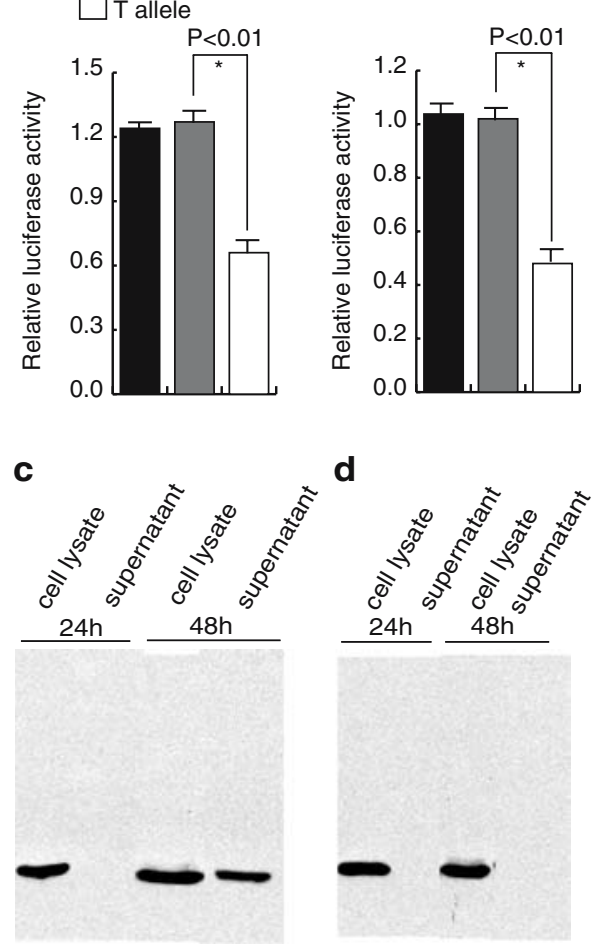

e

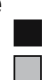

Control siRNA

galectin-2 siRNA
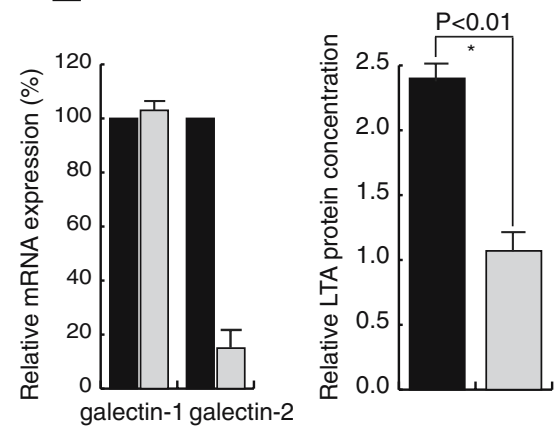

g

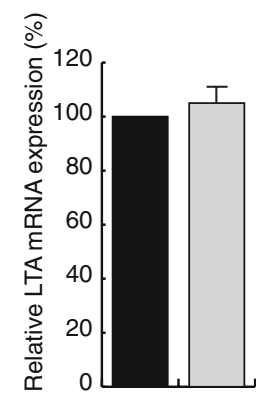

Fig. 4a-g Function of the single nucleotide polymorphism (SNP) in $L G A L S 2$ associated with myocardial infarction (MI) (Ozaki et al. 2004). a, b Transcriptional regulatory activity of intron 1 SNP of $L G A L S 2$ in HeLa (a) and HepG2 (b) cells. c, d Unlike galectin-1 (c), galectin-2 (d) was not a secreted protein. e-g Inhibition of galectin-2 expression. Levels of galectin-1 or galectin-2 mRNA (e), supernatant lymphotoxin- $\alpha(L T A)$ protein (f), and LTA mRNA (g) after $48 \mathrm{~h}$ of transfection with siRNA vectors. The figure was partly cited from Ozaki et al. (2004)

1999). We identified two specific bands that could be seen only when the galectin-2-TAP tag was expressed (Fig. 5a). Based on a MALDI/ToF mass spectrometry analysis, the two bands were identified as $\alpha$ - and $\beta$-tubulins, both constituting microtubules. Using FLAG-tagged galectin-2 transfected HeLa cells, we confirmed co-immunoprecipitation of endogenous tubulins (Fig. 5b). Interestingly, we also found that the 
a

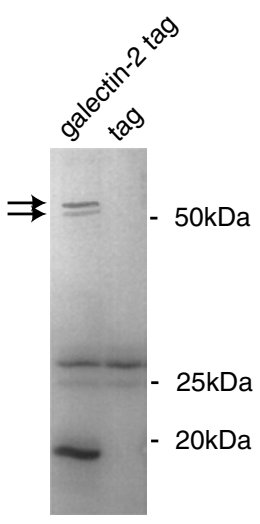

b

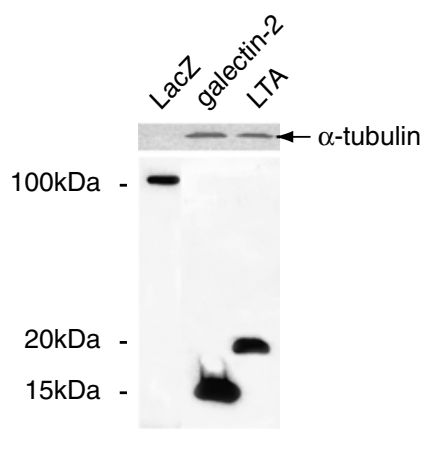

LTA gene

tubulin
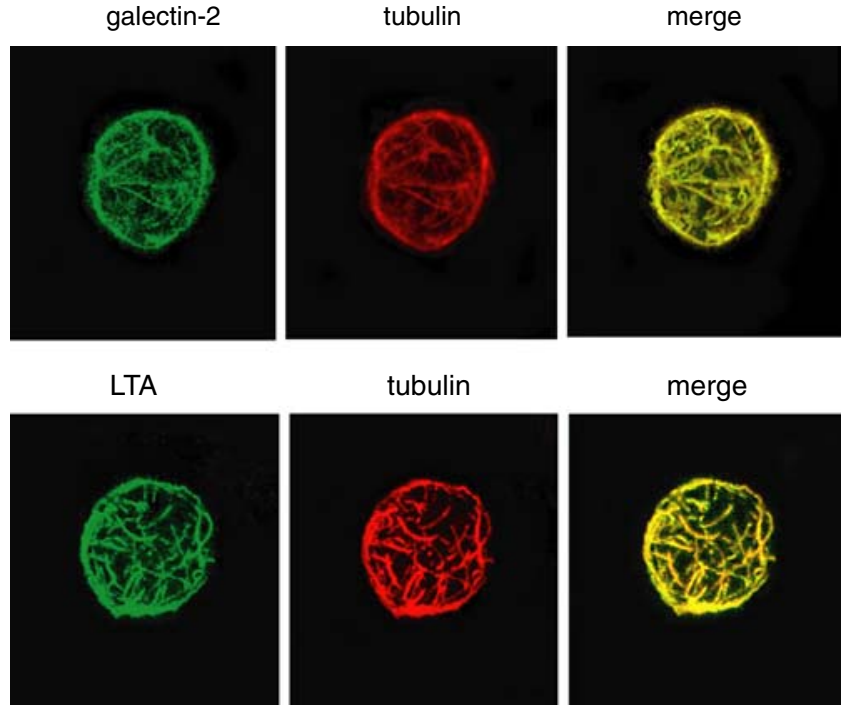

Fig. 5a-c Galectin-2 interacts with microtubules (Ozaki et al. 2004). a Isolation of tandem affinity purification (TAP)-tagged galectin-2 and interacting proteins. Arrowheads indicate $\alpha$ - and $\beta$ tubulins, revealed by MALDI/ToF mass spectrometry analysis. b Co-immunoprecipitation of endogenous $\alpha$-tubulin with FLAGtagged galectin-2 or lymphotoxin- $\alpha$ ( $L T A)$. Immunoprecipitations were done using anti-FLAG M2 agarose, and immunoprecipitates were detected using anti $\alpha$-tubulin antibody (upper panel) or antiFLAG antibody (lower panel). FLAG-tagged LacZ encoding $\beta$ galactosidase was used as a negative control. $\mathbf{c}$ Co-localization of endogenous $\alpha$-tubulin with endogenous galectin- 2 or lymphotoxin$\alpha(L T A)$ in U937 cells. The figure is taken from Ozaki et al. (2004)

endogenous tubulins were also co-immunoprecipitated with LTA (Fig. 5b). Images from serial confocal sections of double-immunostained U937 cells revealed that galectin- 2 and $\alpha$-tubulin co-localized as reticular filamentous networks developed in the cytoplasm (Fig. 5c).

Recently, microtubule cytoskeleton networks have been implicated in the subcellular movements of some proteins including glucose transporter isoform (GLUT4) or thiamine transporter (THTR1) (Liu et al. 2003; Subramanian et al. 2003). It is likely that LTA is another molecule that uses the microtubule cytoskeleton network for translocation, and galectin-2 mediates LTA trafficking through binding to microtubules, although
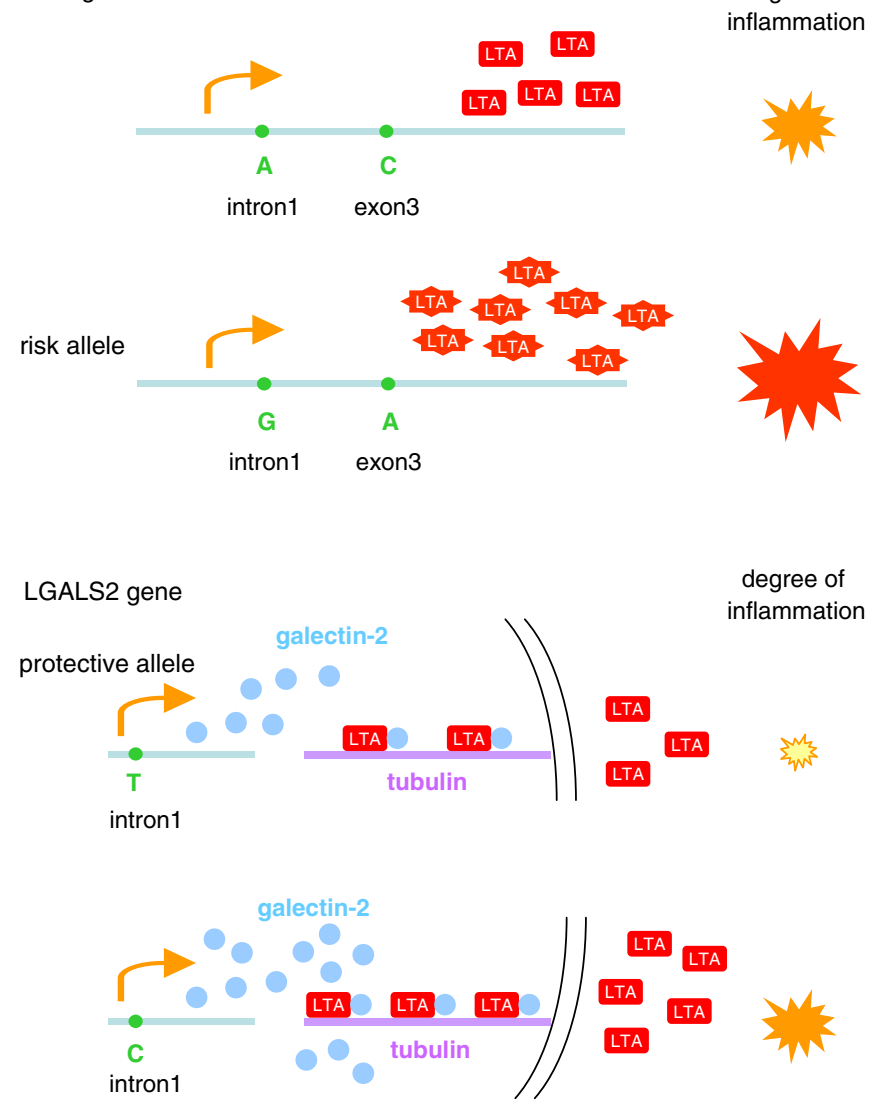

LGALS2 gene

Fig. 6 Hypothetical roles of SNPs in LTA (upper panel) and $L G A L S 2$ (lower panel) in inflammatory process in the pathogenesis of myocardial infarction (MI)

the precise role of galectin-2 in this trafficking machinery complex has yet to be elucidated (Fig. 6).

\section{Other genetic association studies on MI}

By searching the OMIM (Online Mendelian Inheritance in Man; http://www.ncbi.nlm.nih.gov/entrez/query.fcgi?db=OMIM) database with the keyword "myocardial infarction," one can find 132 distinct entries related to this disease. Most of the loci were examined using a small-scale patient group, thus indicating the fragility of their association results. However, even with those that examined a large number of patients, there is scarcely a follow-up study to confirm the association with this disorder. It might be conceivable that the next step should be a prospective confirmatory study and/or meta-analysis.

\section{Molecular analyses on MI regarding inflammation}

There have been studies that reported the importance of inflammation in the pathogenesis of this disorder. For example, rupture of atherosclerotic plaques occurs pre- 
dominantly at the edges of the fibrous cap, an area of accumulated inflammatory cells in close proximity to vascular smooth-muscle cells and endothelial cells (Galis et al. 1994; Jonasson et al. 1986; Kaartinen et al. 1994, 1996; van der Wal et al. 1994). Activated inflammatory cells stimulate their neighboring cells to erode the extracellular matrix through the release of inflammatory cytokines, and decay of the framework forming the plaque cap leads to plaque rupture (Galis et al. 1994; Kaartinen et al. 1996). Also, a recent report demonstrated that LTA was expressed in atherosclerotic lesions in mice and that loss of LTA reduced the size of those lesions; on the other hand, loss of TNF did not alter development of lesions in mice fed an atherogenic diet (Schreyer et al. 2002). These implied that LTA, as one of the mediators of inflammation, along with galectin-2 as a regulator of LTA secretion, might play an important role in the pathogenesis of MI.

\section{Conclusions}

Through a large-scale case-control association study using SNPs, we found two SNPs in LTA confer risk of MI; one in $L G A L S 2$, encoding a binding protein of LTA protein, was also associated with MI. With these two association results as well as recent molecular studies on MI, we believe inflammation, and especially the LTA cascade, may play a pivotal role in the pathogenesis of MI. It is worth noting that these findings initially came from a hypothesis-free, large-scale SNP association study. This indicates the potent power of this kind of study to identify an anchoring point for pathogenesis, and that for further understanding, combination of a search for a binding partner of the encoded protein and a subsequent genetic analysis might also be powerful. It is also possible that a common final pathway that emerges as inflammation is present in the pathogenesis of MI, and due to these characteristics, identification of genetic risk factors of this clinically heterogeneous disorder was relatively easy. Needless to say, this is a speculation and needs to be clarified.

CAD attributable to atherosclerosis is a leading cause of death for both men and women in many countries (Breslow 1997; Braunwald 1997). We believe that knowledge of genetic factors contributing to the pathogenesis of MI will lead to improved diagnosis, treatment and prevention for the patients.

Acknowledgements This work was supported in part by a grant from the Takeda science foundation, the Uehara science foundation and the Japanese Millennium Project.

\section{References}

Braunwald E (1997) Shattuck Lecture - cardiovascular medicine at the turn of the millennium: triumphs, concerns, and opportunities. N Engl J Med 337:1360-1369
Breslow JL (1997) Cardiovascular disease burden increases, NIH funding decreases. Nat Med 3:600-601

Collins FS, Guyer MS, Charkravarti A (1997) Variations on a theme: cataloging human DNA sequence variation. Science 278:1580-1581

den Dunnen JT, Antonarakis SE (2000) Mutation nomenclature extensions and suggestions to describe complex mutations: a discussion. Hum Mutat 15:7-12

Falk E, Shah PK, Fuster V (1995) Coronary plaque disruption. Circulation 92:657-67

Galis ZS, Muszynski M, Sukhova GK, Simon-Morrissey E, Unemori EN, Lark MW, Amento E, Libby P (1994) Cytokinestimulated human vascular smooth muscle cells synthesize a complement of enzymes required for extracellular matrix digestion. Circ Res 75:181-189

Gitt MA, Massa SM, Leffler H, Barondes SH (1992) Isolation and expression of a gene encoding L-14-II, a new human soluble lactose-binding lectin. J Biol Chem 267:10601-10606

Haga H, Yamada R, Ohnishi Y, Nakamura Y, Tanaka T (2002) Gene-based SNP discovey as part of the Japanese Millennium Genome project: identification of 190,562 genetic variations in the human genome. J Hum Genet 47:605-610

Hughes RC (1999) Secretion of the galectin family of mammalian carbohydrate-binding proteins. Biochim Biophys Acta 1473:172-185

Iwanaga Y, Ono K, Takagi S, Terashima M, Tsutsumi Y, Mannami T, Yasui N, Goto Y, Nonogi H, Iwai N (2004) Association analysis between polymorphisms of the lymphotoxin-alpha gene and myocardial infarction in a Japanese population. Atherosclerosis 172:197-198

Jonasson L, Holm J, Skalli O, Bondjers G, Hansson GK (1986) Regional accumulations of $\mathrm{T}$ cells, macrophages, and smooth muscle cells in the human atherosclerotic plaque. Arteriosclerosis 6:131-138

Kaartinen M, Penttila A, Kovanen PT (1994) Accumulation of activated mast cells in the shoulder region of human coronary atheroma, the predilection site of atheromatous rupture. Circulation 90:1669-1678

Kaartinen M, Penttila A, Kovanen PT (1996) Mast cells in ruptureprone areas of human coronary atheromas produce and store TNF-alpha. Circulation 94:2787-2792

Lander ES (1996) The new genomics: global views of biology. Science 274:536-539

Libby P (1995) Molecular bases of the acute coronary syndromes. Circulation 91:2844-2850

Liu LB, Omata W, Kojima I, Shibata H (2003) Insulin recruits GLUT4 from distinct compartments via distinct traffic pathways with differential microtubule dependence in rat adipocytes. J Biol Chem 278:30157-30169

Marenberg ME, Risch N, Berkman LF, Floderus B, de Faire U (1994) Genetic susceptibility to death from coronary heart disease in a study of twins. N Engl J Med 330:1041-1046

Nishimura M, Maeda M, Matsuoka M, Mine H, Saji H, Matsui M, Kuroda Y, Kawakami H, Uchiyama T (2000) Tumor necrosis factor, tumor necrosis factor receptors type 1 and 2, lymphotoxin-alpha, and HLA-DRB1 gene polymorphisms in human T-cell lymphotropic virus type I associated myelopathy. Hum Immunol 61:1262-1269

Ohnishi Y, Tanaka T, Ozaki K, Yamada R, Suzuki H, Nakamura Y (2001) A high-throughput SNP typing system for genomewide association studies. J Hum Genet 46:471-477

Ozaki K, Ohnishi Y, Iida A, Sekine A, Yamada R, Tsunoda T, Sato H, Sato H, Hori M, Nakamura Y, Tanaka T (2002) Functional SNPs in the lymphotoxin-alpha gene that are associated with susceptibility to myocardial infarction. Nature Genet 32:650-654

Ozaki K, Inoue K, Sato H, Iida A, Ohnishi Y, Sekine A, Sato H, Odashiro K, Nobuyoshi M, Hori M, Nakamura Y, Tanaka T (2004) Functional variation in LGALS2 confers risk of myocardial infarction and regulates lymphotoxin-alpha secretion in vitro. Nature 429:72-75 
PROCARDIS Consortium (2004) A trio family study showing association of the lymphotoxin-alpha N26 (804A) allele with coronary artery disease. Eur J Hum Genet 12:770-774

Quasney MW, Bronstein DE, Cantor RM, Zhang Q, Stroupe C, Shike H, Bastian JF, Matsubara T, Fujiwara M, Akimoto K, Newburger JW, Burns JC (2001) Increased frequency of alleles associated with elevated tumor necrosis factor-alpha levels in children with Kawasaki disease. Pediatr Res 49:686-690

Rigaut G, Shevchenko A, Rutz B, Wilm M, Mann M, Seraphin B (1999) A generic protein purification method for protein complex characterization and proteome exploration. Nature Biotechnol 17:1030-1032

Risch N, Merikangas K (1996) The future of genetic studies of complex human diseases. Science 273:1516-1517

Ross R (1999) Atherosclerosis - an inflammatory disease. N Engl J Med 340:115-126

Schreyer SA, Vick CM, LeBoeuf RC (2002) Loss of lymphotoxinalpha but not tumor necrosis factor-alpha reduces atherosclerosis in mice. J Biol Chem 277:12364-12368
Subramanian VS, Marchant JS, Parker I, Said HM (2003) Cell biology of the human thiamine transporter-1 (hTHTR1). Intracellular trafficking and membrane targeting mechanisms. J Biol Chem 278:3976-3984

Tokushige K, Tsuchiya N, Hasegawa K, Hashimoto E, Yamauchi $\mathrm{K}$, Komatsu T, Hayashi N (2003) Influence of TNF gene polymorphism and HLA-DRB1 haplotype in Japanese patients with chronic liver disease caused by HCV. Am J Gastroenterol 98:160-166

van der Wal AC, Becker AE, van der Loos CM, Das PK (1994) Site of intimal rupture or erosion of thrombosed coronary atherosclerotic plaques is characterized by an inflammatory process irrespective of the dominant plaque morphology. Circulation 89:36-44

Yamada A, Ichihara S, Murase Y, Kato T, Izawa H, Nagata K, Murohara T, Yamada Y, Yokota M (2004) Lack of association of polymorphisms of the lymphotoxin alpha gene with myocardial infarction in Japanese. J Mol Med 82:477-483 